\title{
A MODEL OF THE ELECTRON TRANSPORT IN A QUANTUM WIRE WITH CONTACTS
}

\author{
T. JUNGWIRTH
}

Institute of Physics, Czechoslovak Academy of Sciences

$\mathrm{Na}$ Slovance 2, 18040 Prague 8, Czecho-Slovakia

\begin{abstract}
A new model of the electron transport in a quantum wire with contacts is presented. The contact between the reservoir and the quantum part of the system is assumed to be lengthwise the ideal lead. The equilibrium state of electrons in the reservoir gives the final distribution of a non-equilibrium electron system in the ideal lead. The model enables a continuous quasi-1D description of the electron transport evoked by a difference of chemical potentials as well as by a difference of temperatures of reservoirs.
\end{abstract}

PACS numbers: 73.20.Dx

\section{Introduction}

In the studies of the quantum transport in quasi-1D mesoscopic systems (quantum wires) Landauer's scattering approach has been wide spread [1].

Landauer divides the system into three regions: sample, reservoirs and ideal leads (see Fig. 1). The sample is an elastic scattering region whose conductance is

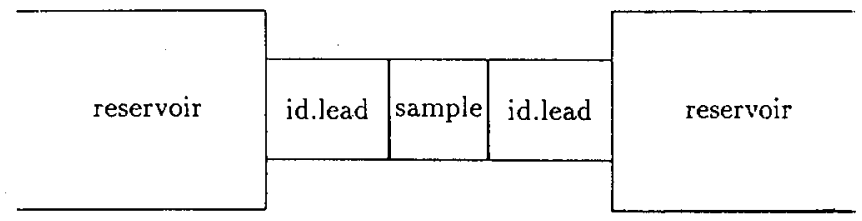

Fig. 1. Landauer's model.

studied. Reservoirs serve as sources and drains of electrons and are the only part where inelastic processes occur. It is assumed that the influence of the current on reservoirs is to be neglected and the electron system in reservoirs is in a thermodynamical equilibrium. A contact between the sample and reservoirs is mediated by ideal leads - perfect wires where no scattering is supposed to exist.

Landauer's approach is based on a complete spatial separation of the regions which leads to the separation between elastic and inelastic scattering. It means 
that the coherent electron transport is investigated since all incoherent processes are hidden in reservoirs. In addition, as the transport doesn't occur in reservoirs a quasi-1D problem is studied.

This simplification, however, leads to difficulties. Due to the current cut off in points separating reservoirs and ideal leads it is not easy to formulate boundary conditions for wave functions in the sample and ideal leads. The problem is usually solved by assuming reservoirs to be only asymptotic parts of the system. Then the states of electrons in reservoirs characterized by chemical potentials $\mu_{\mathrm{L}}, \mu_{\mathrm{R}}$ are taken as boundary conditions.

In this paper we present an alternative model to get a more realistic description of the electron transport in the quantum wire (see Fig. 2). The main idea is

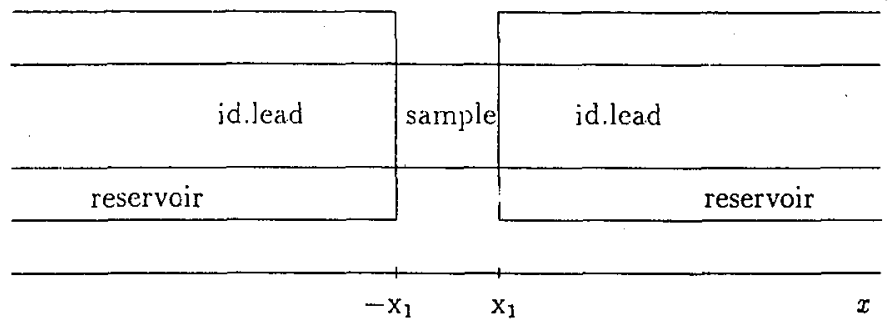

Fig. 2. A new model.

to consider the particle exchange between the reservoir and the quantum wire to be the whole ideal lead lengthwise. (One can imagine the contact like a drop of tin on a wire.) This way the model enables a continuous description of the transport in the whole longitudinal direction.

Because of the position of reservoirs lying on ideal leads we have now generally a 3D problem. Nevertheless it can be reduced to a quasi-1D problem, as shown in the next section.

It is also shown that the model gives new possibilities to investigate the electron transport in quantum wires evoked not only by a difference of chemical potentials but also by a difference of temperatures of reservoirs.

\section{DC-conductance, thermopower}

In this section the behaviour of the model is illustrated and proved by calculating the dc-conductance of the sample and the thermopower. We chose a simple case where a semiclassical description of the transport in the longitudinal direction can be used. In addition we consider the Fermi level of the electron system in the quantum wire and energy levels of the cross quantization corresponding to one open conductance channel.

At first it is necessary to characterize the contact between the reservoir and the quantum wire. We assume that due to the particle exchange the state of electrons in the reservoir gives the final distribution of a non-equilibrium electron system in the ideal lead. As we do not describe each electron transition from the 
reservoir to the ideal lead or back but only the final distribution of the quasi-1D electron system in the ideal lead is defined we solve a quasi-1D transport problem.

Before writing transport equations we make following assumptions:

1. No scattering occurs in the sample and ideal leads, i.e. the ballistic regime of the transport is studied.

2. The particle exchange between reservoirs and ideal leads can be described within the local relaxation time approximation.

3. There is a zero electrostatic field in ideal leads and ideal leads are infinite in the longitudinal direction as in Landauer's approach.

The Boltzmann kinetic equation for a distribution function $f_{\mathrm{C}}(k, x)$ of electrons in the sample reads

$$
\frac{\hbar k}{m} \frac{\partial f_{\mathrm{C}}(x, k)}{\partial x}+\frac{|e|}{\hbar} \frac{\mathrm{d} \Phi}{\mathrm{d} x} \frac{\partial f_{\mathrm{C}}(x, k)}{\partial k}=0,
$$

where $\Phi$ is the potential of the electrostatic field in the sample. Changing variables $k \rightarrow E$ ( $E$ is the total one-electron energy) we obtain [2]

$$
\frac{\hbar k(x)}{m} \frac{\partial f_{\mathrm{C}}(x, E, \pm)}{\partial x}=0,
$$

where $f_{\mathrm{C}}(x, E,+)$ is a distribution function of electrons with $k$ positive and$f_{\mathrm{C}}(x, E,-)$ with $k$ negative and

$$
k^{2}(x)=\frac{2 m}{\hbar^{2}}[E+\Phi(x)] .
$$

In leads the Boltzmann equation is written as

$$
\frac{\hbar k_{\mathrm{L}(\mathrm{R})}}{m} \frac{\partial f_{\mathrm{L}(\mathrm{R})}(x, E, \pm)}{\partial x}=\mp \frac{f_{\mathrm{L}(\mathrm{R})}(x, E, \pm)-f_{\mathrm{L}(\mathrm{R})}^{0}(E)}{\tau},
$$

where $f_{\mathrm{L}(\mathrm{R})}^{0}(E)$ is an equilibrium distribution function corresponding to the electron state in the left (right) reservoir.

Requiring the distribution function to be finite in $\pm \infty$ and matching it in the points $-x_{1}, x_{1}$ we get the solution

$$
\begin{aligned}
& f_{\mathrm{L}}(x, E,+)=f_{\mathrm{C}}(x, E,+)=f_{\mathrm{L}}^{0}(E), \\
& f_{\mathrm{R}}(x, E,+)=f_{\mathrm{R}}^{0}(E)+\left[f_{\mathrm{L}}^{0}(E)-f_{\mathrm{R}}^{0}(E)\right] \exp \left[\frac{m\left(x_{1}-x\right)}{\hbar \tau k_{\mathrm{R}}}\right], \\
& f_{\mathrm{L}}(x, E,-)=f_{\mathrm{L}}^{0}(E)+\left[f_{\mathrm{R}}^{0}(E)-f_{\mathrm{L}}^{0}(E)\right] \exp \left[\frac{m\left(x_{1}+x\right)}{\hbar \tau k_{\mathrm{L}}}\right], \\
& f_{\mathrm{C}}(x, E,-)=f_{\mathrm{R}}(x, E,-)=f_{\mathrm{R}}^{0}(E) .
\end{aligned}
$$

Without calculating the exact expression for the current one can see the consequence of the particle exchange between reservoirs and leads. The current in the sample does not depend on the $x$-coordinate while in leads it exponentially decreases to a zero value.

Now we'll derive the expression for the electrical conductance and discuss the effect of the difference of reservoir temperatures. In both cases we need a relation 
between the current and the distribution function. Full form should be written in one dimension as:

$$
j=\frac{\hbar e}{\pi m} \int_{-\infty}^{\infty} k f \mathrm{~d} k
$$

\subsection{The electrical conductance}

The electrical dc-conductance is calculated at the zero temperature as in works based on Landauer's approach. The equilibrium distribution functions for $T=0 \mathrm{read}$

$$
f_{\mathrm{L}(\mathrm{R})}^{0}\left(E \leq E_{\mathrm{Fermi}}^{\mathrm{L}(\mathrm{R})}\right)=1
$$

and

$$
f_{\mathrm{L}(\mathrm{R})}^{0}\left(E>E_{\mathrm{Fermi}}^{\mathrm{L}(\mathrm{R})}\right)=0 .
$$

Then

$$
j=\frac{e^{2}}{\pi \hbar} U
$$

where $U \equiv E_{\text {Fermi }}^{\mathrm{L}}-E_{\text {Fermi }}^{\mathrm{R}}$.

For the one-channel conductance we obtain the well-known expression

$$
G \equiv \frac{j}{U}=\frac{e^{2}}{\pi \hbar} \text {. }
$$

\subsection{The thermopower}

To describe the effect of the temperature difference of reservoirs we derive the expression for the voltage compensating the effect. The case of $T_{R}>T_{L}$ is considered.

For non-zero temperatures of reservoirs the equilibrium distribution functions are

$$
f_{\mathrm{L}(\mathrm{R})}^{0}(E)=1 /\left\{1+\exp \left[\frac{\left(\hbar^{2} k^{2} / 2 m-\mu_{\mathrm{L}(\mathrm{R})}\right)}{\left(k_{\mathrm{B}} T_{\mathrm{L}(\mathrm{R})}\right)}\right]\right\}
$$

Then

$$
j=\frac{e}{\pi \hbar}\left\{\mu_{\mathrm{L}}-\mu_{\mathrm{R}}+\ln \frac{\left[1+\exp \left(-\mu_{\mathrm{L}} /\left(k_{\mathrm{B}} T_{\mathrm{L}}\right)\right)\right]^{k_{\mathrm{B}} T_{\mathrm{L}}}}{\left[1+\exp \left(-\mu_{R} /\left(k_{\mathrm{B}} T_{\mathrm{L}}\right)\right)\right]^{k_{\mathrm{B}} T_{\mathrm{R}}}}\right\} .
$$

Demanding $j$ to be equal to zero we obtain the compensating voltage (defined as $U \equiv\left(\mu_{\mathrm{L}}-\mu_{\mathrm{R}}\right) /|e|$

$$
U=-\frac{k_{\mathrm{B}} T_{\mathrm{R}}}{|e|} \ln \left\{\left[1+\exp \left(-\frac{\mu_{\mathrm{L}}}{k_{\mathrm{B}} T_{\mathrm{L}}}\right)\right]^{T_{\mathrm{L}} / T_{\mathrm{R}}}-\exp \left(-\frac{\mu_{\mathrm{L}}}{k_{\mathrm{B}} T_{\mathrm{R}}}\right)\right\} .
$$

It is easy to find that the expression for the voltage complies with conditions $U>0$ for $T_{\mathrm{R}}>T_{\mathrm{L}}$ and $U=0$ for $T_{\mathrm{R}}=T_{\mathrm{L}}$. 


\section{Summary}

A new more realistic model of the electron transport in the quantum wire is presented in this paper. As shown the new model avoids difficulties following from Landauer's approach in formulating boundary conditions. A trivial condition of the finite value of the distribution function in $\pm \infty$ is sufficient.

Proving the model by calculating the electrical dc-conductance we found that the well-known expression $e^{2} / \pi \hbar$ can be obtained from semiclassical (Boltzmann) transport equations. As shown on the example of the thermopower the great advantage of the model is that it should be useful in studies of more complicated transport problems in quasi-1D mesoscopic systems.

\section{Acknowledgments}

The author is indebted to his supervisor B. Velický for all the time he devoted. He also wishes to thank J. Mašek for helpful discussions and for a critical reading of the manuscript.

\section{References}

[1] R. Landauer, IBM J. Res. Dev. 1, 223 (1957).

[2] G.D. Mahan, J. Appl. Phys. 57, 2242 (1985).

[3] B. Velický, V. Spička, J. Mašek, Solid State Commun. 72, 981 (1989).

[4] I.B. Levinson, Zh. Eksp. Teor. Fiz. 95, 2175 (1989). 